\title{
MERLIN and EVN Observations of a $z=4.514$ Radio Galaxy, RC J0311+0507, and the problem of SMBH's in the Early Universe
}

\section{Yu.N. Parijskij, O.P. Zhelenkova*, A.I. Kopylov, O.V. Verkhodanov, T.A. Fatkhullin} Special Astrophysical Observatory of RAS, Niznij Arkhyz, 369167, Russia E-mail: zhedsao.ru (O.P. Zhelenkova)

\section{P. Thomasson, T.W.B. Muxlow, R. Beswick}

The University of Manchester, JBO, Macclesfield, Cheshire SK11 9DL, U.K. E-mail: ptejb.man.ac.uk (P.Thomasson)

\section{N.S. Soboleva, A.V. Temirova}

SPb Branch of SAO RAS, Pulkovskoe sh., 65, Saint Petersburg, 196140, Russia E-mail: sns33@mail.ru (N.S. Soboleva)

\begin{abstract}
RC J0311+0507, the parent galaxy of which has a redshift, $\mathrm{z}$, of 4.514, is one of the most luminous objects in the High-z radio Universe. New MERLIN and EVN intensity and polarization maps of the object at $1.7 \mathrm{GHz}$ and $5 \mathrm{GHz}$ show multiple compact components and extended emission orientated in a primarily NS direction on either side of what appears to be a core. The flux density ratio between the southern and northern components is highly asymmetric, with values of $\sim 30$ at $5 \mathrm{GHz}$ and $\sim 10$ at $1.7 \mathrm{GHz}$. New optical observations using the Russian 6-m optical telescope confirm the identification of the radio source with a galaxy, and show the presence of extended $L y_{\alpha}$ emission. The very high radio luminosities and the high "bulge optical emission" suggest the presence of a very massive $\left(10^{9} M_{\odot}\right)$ Black Hole $(\mathrm{BH})$. Assuming that the age of the Universe at $\mathrm{z}=4.514$ is only $\sim 1.3 \mathrm{Gyr}$ and that the standard epoch for the formation of galaxies is within the redshift range from 7 to 10 , there is less than $0.5 \mathrm{Gyr}$ available for the formation of RC $\mathrm{J} 0311+0507$. The quick growth of a BH mass solely as a result of a merger is questionable, and the simple evolution from several solar masses to $10^{9} M_{\odot}$ in a timescale of less $1 \mathrm{Gyr}$ appears to be very improbable.
\end{abstract}

10th European VLBI Network Symposium and EVN Users Meeting: VLBI and the new generation of radio arrays

September 20-24, 2010

Manchester UK

${ }^{*}$ Speaker. 

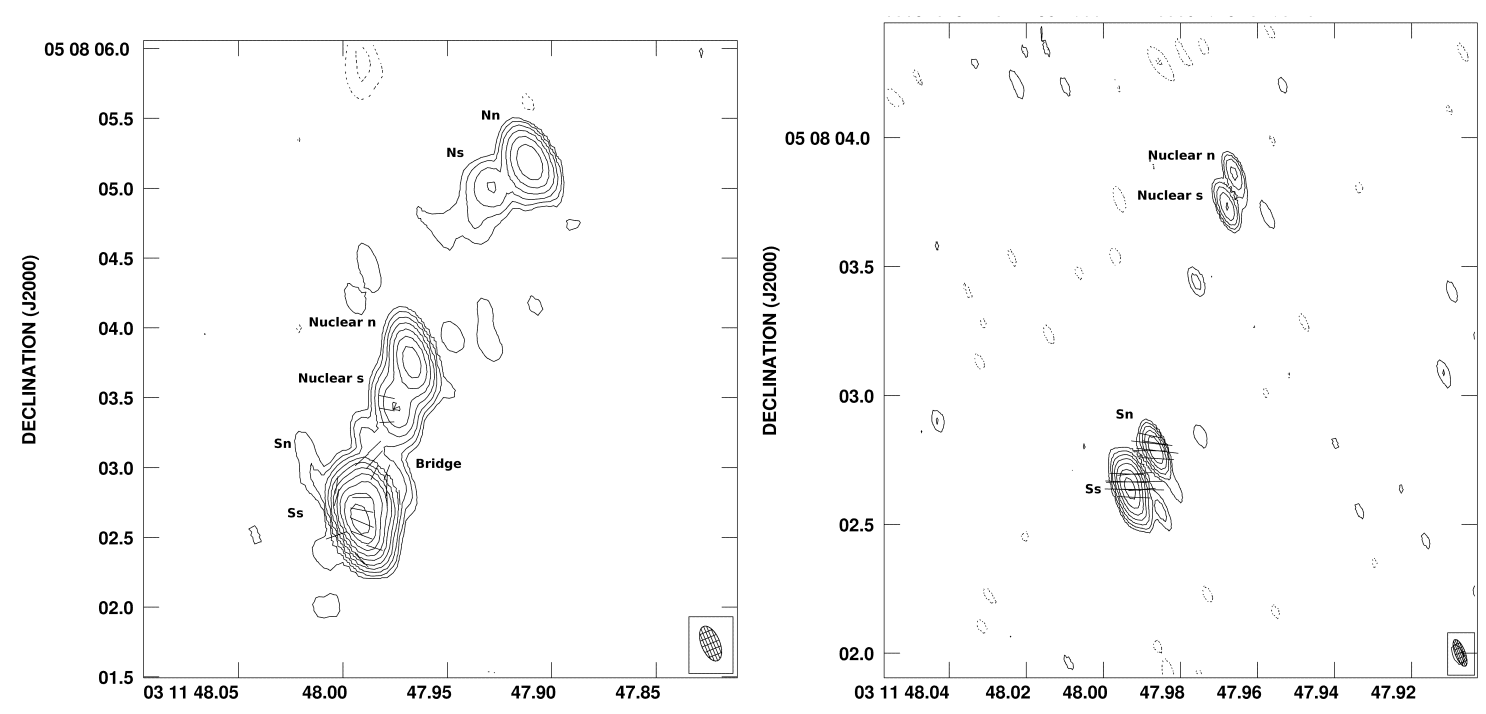

Figure 1: The $\mathrm{z}=4.514$ radio galaxy, RC J0311+0507, images: MERLIN $1.7 \mathrm{GHz}$ (left) and MERLIN $5 \mathrm{GHz}$ (right) with restoring beams $0.264^{\prime \prime} \times 0.132^{\prime \prime}$ and $0.110^{\prime \prime} \times 0.039^{\prime \prime}$, respectively.

\section{Introduction}

RC J0311+0507 was recognized as having an Ultra Steep Spectrum (USS) $\alpha=-1.31\left(S \propto v^{\alpha}\right)$ between $365 \mathrm{MHz}$ and $4850 \mathrm{MHz}$ in the early stages of the "Big Trio" survey project (RATAN-600 as the finding survey instrument, the VLA as the imaging instrument, and the Russian 6-m optical telescope for photometry and spectroscopy) [1]-5]. This survey was based on the earlier RATAN-600 1980 "Cold" radio survey at $7.6 \mathrm{~cm}$ [6-8]. An initially unpublished version of the catalogue derived from the UTRAO survey at $365 \mathrm{MHz}$ [9] helped to provide the spectral index information and a necessary better position for the VLA observations. The only known object close to the position of RCJ0311+0507 was 4C+04.11, and a first VLA image of it at $1425 \mathrm{MHz}$ suggested an unequal double structure and the object was classified as a candidate USS, FRII [10] radio galaxy. Further observations with the VLA [2] at resolutions of $4.5^{\prime \prime}$ and $0.5^{\prime \prime}$ at $1425 \mathrm{MHz}$ and $4860 \mathrm{MHz}$ respectively showed an asymmetrical double structure for this compact steep spectrum (CSS) object. Optical photometric and spectroscopic observations with the Russian 6-m telescope showed the presence of a strong emission line at $6703 \AA$ in what was possibly the host galaxy, which was observable as a $23^{m}$ object in $\mathrm{R}$ band. This emission line was identified with $L y_{\alpha}$ at a redshift of $\mathrm{z}=4.514$ [四]. This redshift was recently confirmed by observations with the SINFONI spectrograph installed on UT4 of the VLT [11].

\section{Radio and optical observations}

RC J0311+0507 was imaged with MERLIN at $1.7 \mathrm{GHz}$ (Fig. 1, left) and $5 \mathrm{GHz}$ (Fig. 1, right). A more complex structure was seen than in earlier observations, but with the core of the radio emission still unidentifiable. The nature of the compact components, whether hotspots as part of a double-double structure or knots along jets in the source, were also indeterminable and a MERLIN + VLA image, showing more clearly both the compact and more extended features, did 
not clarify the situation. Consequently, MERLIN and e-VLBI observations (including the Lovell telescope) with a resolution of $0.025^{\prime \prime}$ at $1.7 \mathrm{GHz}$ were undertaken (Fig. 2).

Following the initial MERLIN observations and results, new optical observations in broad I and middle SED 665 filters were made with the 6-m telescope in a direct imaging mode using SCORPIO [12] with $1.2^{\prime \prime}$ to $1.5^{\prime \prime}$ seeing conditions (Fig. 3, left). All the R-band available observations (4 separate epochs) were used to improve the astrometry.

\section{Results}

The MERLIN and e-VLBI L-Band images clearly show that the source has an FRII structure, but with a very high asymmetry in the flux densities of the northern and southern components $(\sim 30$ at $5 \mathrm{GHz}$ and $\sim 10$ at $1.7 \mathrm{GHz}$ ). In fact, these are close to the extreme of what has been seen for radio galaxies. There appear to be 8 small-sized, individual components in the structure, which are numbered in Fig. 2. There is a jet from an unresolved component (3) pointing to the south which follows a highly curved path, particularly as it approaches the hotspot region. Immediately prior to the southern hotspots $(7 \& 8)$, the jet appears to be on the outer edge of a region of weak, but extended emission at the noise level of the image. The curvature of the jet could arise if it is moving through a cluster medium. The extent of the radio source $\left(\sim 2.7^{\prime \prime}\right)$ appears to be approximately the same as that of the optically visible galaxy, but this could be illusory if the orientation of the radio source relative to us is such that it is pointing almost directly towards or away from us.

The southern hot-spot regions show a mean polarization at $5 \mathrm{GHz}$ of $\sim 12 \%(25 \%$ and $8.6 \%$ for components 7 and 8 respectively), with somewhat less polarization at L-Band $(\sim 10 \%)$. These values are typical for the hot-spot regions in FRII radio sources. The fact that the polarization angles do not change appreciably indicates that a regular magnetic field dominates over a turbulent one, which limits any "internal" rotation measure. Further evidence for this is that in the "bridge region" in the MERLIN $1.7 \mathrm{GHz}$ image (Fig. 1, left). The position angle of the polarization is not too dissimilar. Using values of the component angular sizes derived from an image obtained solely from the EVN data, it is possible to make estimates of "equipartition" magnetic fields for all the components. Values between $\sim 0.4$ and $1 \mathrm{mG}$ have been found. These are quite strong fields, which will reduce the life-time of the high energy electrons in the components. The fields are also greater than would be expected from CMB radiation magnetic fields at $\mathrm{z}=4.514$. The reduction in polarization at the lower frequency is probably caused by greater Faraday rotation. There is no measured polarization in the northern lobe / hot-spot region (components $1 \& 2$ ). This could be the result of increased Faraday rotation arising from an increased path length if this region is on the far side of the source from us.

This might be expected from the overall structure, with a southern "beamed" jet pointing towards us and no jet visible to the north of component 3, which appears to be the core. However, it could be that any polarization is barely detectable because of the weakness of the total flux density of the two components. Where possible the spectral indices of the components have been calculated, all of which appear to be steep, with component 1 being the steepest with $\alpha=-2.4$. The most compact (3), has the flattest spectrum with $\alpha=-0.8$, which is much flatter than the value of -1.31 for the integrated spectrum. This would appear to confirm that component 3 is or contains the nuclear source or core. The measured position of this core is: $\alpha_{J 2000}=3^{h} 11^{m} 47.966^{s}$, 
$\delta_{J 2000}=+05^{\circ} 08^{\prime} 03.87^{\prime \prime}$. This is in remarkable agreement with the optical position obtained from a weighted mean of the R-band images of the galaxy, which is: $\alpha_{J 2000}=03^{h} 11^{m} 47.966^{s} \pm 0.008^{s}$, $\delta_{J 2000}=+05^{\circ} 08^{\prime} 03.79^{\prime \prime} \pm 0.16^{\prime \prime}$.

The sizes of the I and SED 665 images are definitely greater than the point spread function, indicating that the parent optical object is a galaxy and not a QSO. In the SED 665 filter, the size of the object is greater than that in the other filters (Fig. 3, left) because of intense hydrogen $L y_{\alpha}$ line radiation, probably arising from gas surrounding the radio galaxy.

\section{Discussion}

The MERLIN and EVN observations have demonstrated that RC J0311+0507 is not a simple double, or a double-double radio galaxy, but has a multi-component structure, each component having a very high luminosity. Its structure appears to be similar to that of the radiogalaxy, 1138-262, observed by Pentericci et al.,

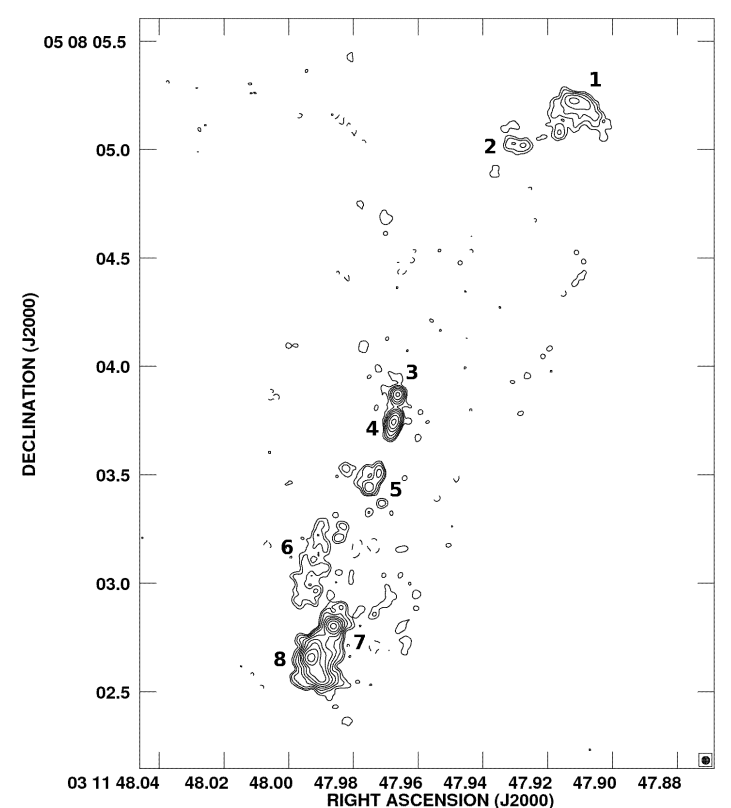

Figure 2: A MERLIN and e-VLBI $1.7 \mathrm{GHz}$ combined image with a restoring beam of $0.035^{\prime \prime} \times 0.035^{\prime \prime}$. 1997 [13], but the latter has a significantly lower redshift of $\sim 2.2$. Both have highly asymmetric flux density distributions, with the visible jet/ hot-spot structure being considerably bent. All components in the sources appear to have steep spectra, with the magnitudes of the spectral indices increasing with distance from the source centres. As indicated above, the lack of measured polarization in the northern lobe of RC J0311+0507 may not be caused by Faraday rotation, but if it is, this is also found for the component on the opposite side of the nucleus from the jet / hot-spot structure in the image of 1138-262. Likewise, one cannot say for certain that the direction of the magnetic field is along the direction of the jet because of orientation effects, but this also appears to be the case as for 1138-262.

RCJ0311+0507 is clearly one of the most powerful radio galaxies at high redshift with a radio luminosity equaling that of the most distant radio galaxy, TN J0924-2201, at a redshift $\mathrm{z}=5.19$ [15] at the same frequency (see [14]). In fact, there are only two radio galaxies (including TN J0924-2201) with such high radio luminosity at $\mathrm{z}>3$, both of which have steeper spectral indices than RC J0311+0507 and have equal luminosities at $400 \mathrm{MHz}$ [15] . Consequently, at shorter centimetre wavelengths, the radio luminosity of RCJ0311+0507 is a factor 2 greater than that of TN J0924-2201 because of its "flatter" spectrum, making it the most luminous galaxy at these wavelengths. A value for the monochromatic luminosity for the nuclear component (3) can be obtained from the MERLIN map at $5 \mathrm{GHz}$, which is $\sim 5 \cdot 10^{26} \mathrm{~W} \cdot \mathrm{Hz}^{-1}$. This is a small fraction $(\sim 1 \%)$ of the integrated radio luminosity at $5 \mathrm{GHz}$, which does not differ significantly from normal values for radio galaxies, but is smaller than would be expected for QSOs. 

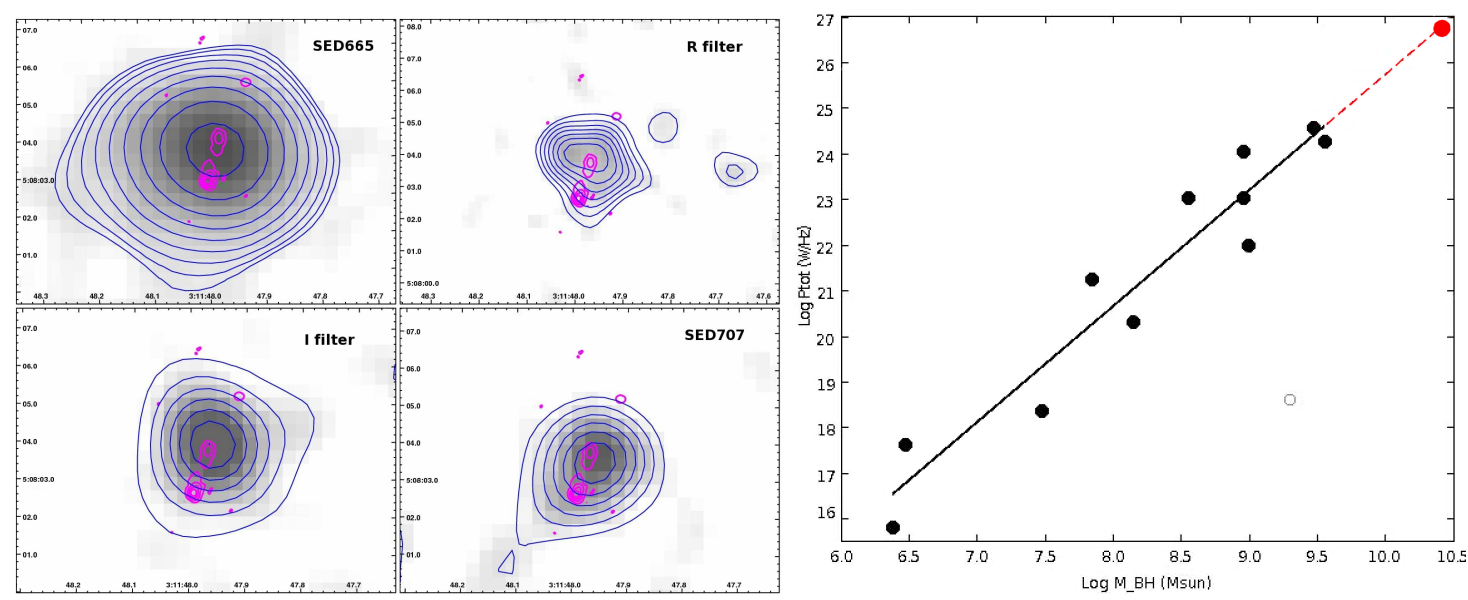

Figure 3: Left: the RC J0311+0507 images (grayscale images with blue contours) in two wide (R and I) and two middle (SED 665 and SED 707) filters with overlaid contours (magenta) of the $5 \mathrm{GHz}$ combined MERLIN and VLA map. Right: an estimation (marked by a red circle) of RC J0311+0507 BH mass from the correlation [16] between total luminosity $P_{t o t}$ at $5 \mathrm{GHz}$ and $\mathrm{BH}$ mass.

According to the well known correlations between integrated radio luminosities, $P_{t o t}$, and BH mass [17], $\left(P_{t o t} \propto M_{B H}^{2.5}\right)$ the high total and core radio luminosities and the high bulge optical emission [18] suggest the presence of a very massive BH with a mass greater then $10^{9} M_{\odot}$ in the parent galaxy nucleus. Although the MERLIN and EVN image indicates that the core of the galaxy is located within component 3 , the mass of the $\mathrm{BH}$ is difficult to estimate from this because the flux density of the "flat-spectrum" component within this core region is as yet unknown. However, an estimation of the BH mass can be made from the "remarkably tight correlation" between BH mass and the $5 \mathrm{GHz}$ total radio emission as shown by [17]. Reproducing their plot (3), right), but including RC J0311+0507 (marked by the red circle), its BH mass is seen to be greater then $10^{10} M_{\odot}$, which is close to the present day record. Accepting this value, standard accretion theory would indicate a primordial $\mathrm{BH}$ mass much greater than $10^{3} M_{\odot}$. The alternative is an unusual mechanism for the development of black holes in the early Universe, since the simple evolution from several solar masses to $10^{10} M_{\odot}$ in less than a $1 \mathrm{Gyr}$ time scale looks very improbable. In fact, primordial black holes with masses of at least $10^{3}-10^{5} M_{\odot}$ have been suggested by Wang et al., 2007 [19]. The age of the Universe at $\mathrm{z}=4.514$ is only $\sim 1.3 \mathrm{Gyr}$. Assuming a standard epoch of galaxy formation to be at a redshift of $7-10$, then less than $0.5 \mathrm{Gyr}$ are available for the formation of an object with such an extremely high radio luminosity as RC J0311+0507. From the BVRI photometry, the "nuclear age" of the parent galaxy of RC J0311+0507 has been estimated to be $\sim 0.5 \mathrm{Gyr}$. However, IR data are required to improve these estimates. The "dynamical age" for RC J0311+0507 has not yet been measured, but using the mean dynamical ages for field galaxies in the Hubble Ultra-Deep Field [20] for $z=4.514$, the age of the parent galaxy is expected to be less than $0.3-0.1 \mathrm{Gyr}$, if the giant elliptical were formed by a "merging tree" process from small field galaxies. Even assuming an age of $\sim 0.3 \mathrm{Gyr}$, the parent galaxy must be formed very quickly. There are two possibilities: independent early formation of giant stellar systems with absolute magnitudes $-22^{m}$ before field galaxies, or the independent formation of a SMBH long before the epoch of formation of field galaxies. 
Multi-component radio structures for low luminosity objects can usually be explained by irregular "cooling flows" or by "large molecular cloud" accretions [15]. High luminosity RGs with complex structures and high BH masses could result from mergers of cluster galaxies or from collisions between two SMBHs, see [21, 22]. However, for young, multi-component, very powerful radio galaxies, the usual "multi-merging" explanation seems difficult because of the short time scale for nuclear events - smaller than the free-fall time or galaxy rotation period. Double core or double SMBH variants, discussed recently in Liu \& Wu (2002) [23] may help to resolve problem.

\section{Conclusion}

New MERLIN and EVN data have shown the complex structure of the radio galaxy, RC J0311+0507, the most powerful yet known at short centimetre wavelengths in the Early Universe. The precise nature of the small size sub-components is not clear, but it is possible that nuclear source activity has been stimulated by multi-merging effects in a cluster of galaxies. The age of the parent galaxy has been estimated to be only $\sim 0.5 \mathrm{Gyr}$, but the nature and age of the parent galaxy may be better understood if there were high resolution $\left(0.1^{\prime \prime}\right)$ optical data in all possible spectral bands. Deep ( $>25^{m}$ in R, I) observations would provide a direct confirmation of the existence of a cluster of galaxies at such an early epoch.

\section{Acknowledgments}

This work was supported by grants of the Russian Funds of Basic Researches 08-02-4863a, 07-02-12055ofi, 09-02-12169ofn, 10-07-00412a.

\section{References}

[1] A.I. Kopylov et al., RC J0311+0507: A candidate for superpowerful radio galaxies in the early universe at redshift $z=4.51$, AstL, 32 (433) 2006.

[2] W.M. Goss et al., Investigation of the RATAN-600 steep-spectrum $(\alpha>1.1)$ radio source sample - VLA observations and optical identifications, AZh, 69, (673) 1992.

[3] Yu.N. Parijskij et al., Investigation of RATAN-600 RC radio sources, BSAO, 40 (5) 1996.

[4] Yu.N. Parijskij et al., RATAN-600-VLA-BTA-6m ("Big Trio") project: Multicolor study of distant FRII radio galaxies, $A \& A T, 19$ (297) 2000.

[5] N.S. Soboleva et al., RC J0105+0501: A Radio Galaxy with Redshift z 3.5, AstL, 26 (723) 2000.

[6] A.B. Berlin et al., A deep sky survey at $7.6 \mathrm{~cm}$ with the RATAN-600 radio telescope, PAZh, $\mathbf{5 8}$ (85) 1981.

[7] Yu.N. Parijskij et al., The RATAN-600 $7.6 \mathrm{~cm}$ catalog of radio sources from "Experiment Cold-80", A\&AS, 87 (1) 1991.

[8] Yu.N. Parijskij et al., The RATAN-600 $7.6 \mathrm{~cm}$ catalogue of radio sources within the interval $22^{h}-4^{h}$ at declination of SS433, A\&AS, 96 (583) 1992.

[9] J.N. Douglas et al., The Texas Survey of Radio Sources Covering $-35.5^{\circ}<\delta<71.5^{\circ}$ at $365 \mathrm{MHz}$, AJ, 111 (1945) 1996. 
[10] B.L. Fanaroff \& J.M. Riley, The morphology of extragalactic radio sources of high and low luminosity, MNRAS, 167 (31) 1974.

[11] C. de Breuck, private communication, 2009.

[12] V.L. Afanasiev \& A.V. Moiseev, The SCORPIO Universal Focal Reducer of the 6-m Telescope, PAZh, 31 (214) 2005.

[13] L. Pentericci et al., The radio galaxy 1138-262 at $z=2.2$ : a giant elliptical galaxy at the center of a proto-cluster?, A\&A, 326 (580) 1997.

[14] W. van Breugel et al., A Radio Galaxy at Z = 5.19, ApJ, 518 (L61) 1999.

[15] G. Miley \& C. de Breuck, Distant radio galaxies and their environments, A\&ARv, 15 (67) 2008.

[16] A. Franceschini, S. Vercellone and A.C. Fabian, Supermassive Black Holes in Early-Type Galaxies: Relationship with Radio Emission and Constraints on the Black Hole Mass Function, MNRAS, 297 (817) 1998.

[17] P. Salucci et al., Mass function of dormant black holes and the evolution of active galactic nuclei, MNRAS, 307 (637) 1999.

[18] P.J. McCarthy, High redshift radio galaxies, ARA\&A, 31 (639) 1993.

[19] J.M. Wang et al., Suppressed Star Formation in Circumnuclear Regions in Seyfert Galaxies, ApJ, $66 \mathbf{1}$ (143) 2007.

[20] N.P. Hathi et al., Surface Brightness Profiles of Composite Images of Compact Galaxies at z 4-6 in the Hubble Ultra Deep Field, AJ, 135 (156) 2008.

[21] V. Begelman, R.D. Blandford \& J. Rees, Massive black hole binaries in active galactic nuclei, Nature, 287 (307) 1980.

[22] M. Sikora, L. Stawarz \& J.-P. Lasota, Radio-loudness of active galaxies and the black hole evolution, NewAR, 51 (891) 2008.

[23] F.K. Liu \& X.-B. Wu, Black hole mass and binary model for BL Lac object OJ 287, A\&A, 388 (L48) 2002. 